\title{
Smart Response Surface Models using Legacy Data for Multidisciplinary Optimization of Aircraft Systems
}

\author{
Ramesh Gabbur \\ Scientist,Aeronautical Development Agency,Bangalore.India Doctoral Student at International Institute for \\ Aerospace Engineering \& Management, Jain University, Bangalore.
}

\begin{abstract}
One of the key challenges in multidisciplinary design is integration of design and analysis methods of various systems in design framework. To achieve Multidisciplinary Design Optimization (MDO) goals of aircraft systems, high fidelity analysis are required from multiple disciplines like aerodynamics, structures or performance. High Fidelity Analysis like Computer-Aided Design and Engineering (CAD/CAE) techniques, complex computer models and computation-intensive analyses/simulations are often used to accurately study the system behaviour towards design optimization. Due to high computational cost and numerical noise associated with these analyses, they cannot be used effectively. The use of surrogates or Response Surface Models (RSM) is one approach in Multi Disciplinary design optimization to avoid the computation barrier and to take care of artificial minima due to numerical noise. This paper brings out a method based on use of "Smart Response Surface Models" to generate surrogate models, with its validated subspace, in the design space around the point of interest with the use of legacy data for MDO. The method has been evaluated on three test cases, which are created based on High Speed Civil Transport (HSCT) Multidisciplinary Design Optimization Test Suite
\end{abstract}

Keywords: Optimization, DOE, Surrogate Modelling, Multidisciplinary Design, Aircraft

\section{Introduction}

Present generation multi-role combat aircraft with y by wire and state of the art weapons systems are complex systems in nature, which need specialists. Complexity of combat aircraft mandates the need for design teams to have multidisciplinary experience in the entire aircraft design with core expertise in their respective domains. Today aerospace design and development is not only multidisciplinary but also global in nature with design and engineering teams deployed around the world [1]. It requires a high level of technical and technomanagerial expertise across various engineering disciplines to cater for very stringent reliability, safety and performance requirements. This would enable design and development of an optimal multidisciplinary system in a collaborative and cohesive integrated environment of various engineering domains.

Multidisciplinary system design is a complex, computationally intensive process that combines discipline analysis with design-space search and decision making. The decision making is based on engineering judgment and is greatly assisted by computer automation. Towards this systems engineering provides holistic approach for integrated design and development of aircraft and its associated systems [2]. One of the key challenges in collaborative design is integration of design and analysis methods of various systems in system engineering framework. With the advances in Computer-Aided Design and Engineering (CAD/CAE) techniques, complex computer models and computation-intensive analyses/simulations (discipline analysis) are often used to accurately study the system behaviour towards design improvements. This design optimization process normally requires a large number of iterations before the optimal solution is identified. Design optimization, with high fidelity design tools, is computationally very expensive and time consuming. The use of approximation models or surrogates to replace the expensive high fidelity computer analysis, in Multi Disciplinary Optimization (MDO), is a natural approach to avoid the computation barrier and to take care of numerical noise[3]. Typically approximation models or surrogates of high fidelity design tools are used to reduce this computational effort and time during multidisciplinary design optimization process. This paper brings out a method based on use of "Smart Response Surface Models" to generate surrogate models in the design space around the point of interest with the use of legacy data for (MDO).

\section{Response Surface Models (Rsm)}

Complex aircraft engineering design problems are solved using high fidelity analysis/simulation software tools. The high computational cost associated with these analyses and simulations prohibits them from being used as performance measurement tools in the optimization of design for combat aircraft. Another major drawback in using high fidelity analysis is numerical noise, which occurs as a result of the incomplete convergence of iterative processes, the use of adaptive numerical algorithms, round-o_errors, and the discrete representation of continuous physical objects (fluids or solids)[4]. The use of surrogates or Response Surface 
Models (RSM) to replace the expensive high fidelity computer analysis, in MDO, is a natural approach to avoid the computation barrier and to take care of artificial minima due to numerical noise. Renaud and Gabriele developed Response Surface Modelling (RSM) of multidisciplinary systems during concurrent subspace optimizations (CSSOs) [5] [6]. Korngold and Gabriele addressed discrete multidisciplinary problems using the RSM [7].

Expensive high fidelity computer analysis can be represented as a blackbox function. In a simplest form the high fidelity analysis tools takes vector $\mathrm{X}$ as input and gives $\mathrm{Y}$ as the output as shown in Figure 1.

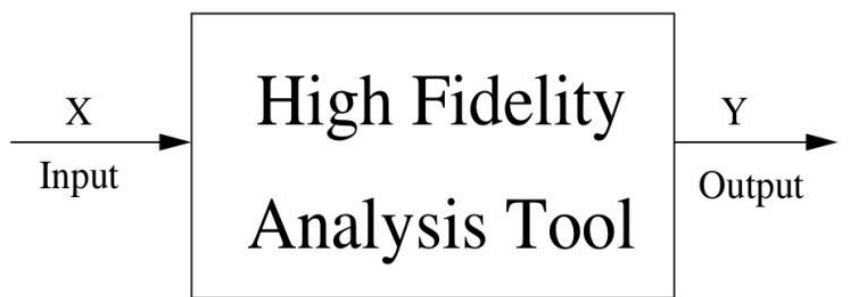

Figure 1: Representing it mathematically with limits on the design space

$$
\begin{aligned}
& Y=f(\bar{x}) \quad \text { where } \bar{x} \in R^{n} \\
& \bar{x}_{\text {min }}<\bar{x}<\bar{x}_{\text {max }} \text { defines the design space }
\end{aligned}
$$

This function would be replaced by polynomial based surrogate model. A typical second order surrogate model is shown below

$$
\begin{aligned}
& y=\beta_{0}+\sum_{i=1}^{n} \beta_{i} x_{i}+\sum_{i=1}^{n} \beta_{i i} x_{i}^{2}+\sum_{i=1}^{n-1} \sum_{j=i+1}^{k n} \beta_{i j} x_{i} x_{j} \\
& \bar{x}_{\text {min }}^{\prime}<\bar{x}^{\prime}<\bar{x}_{\text {max }}^{\prime} \quad \text { defines the model subspace }
\end{aligned}
$$

Where $\beta_{i}, \beta_{j}$ and $\beta_{i j}$ are regression coefficients, $\bar{x}$ is the input vector and $\mathrm{y}$ is the output. The subspace surrogate model is defined by the side constraints $\bar{x}_{\text {min }}^{\prime}$ and $\bar{x}_{\text {max }}^{\prime}$.

\section{Smart Response Surface Models}

Smart response surface models is a methodology that develops a response surface model and identifies the subspace for which model is valid. In the conventional methods, of implementing response surface models (RSM) for Multidisciplinary Design, the model subspace is de_ned prior to generating the model and the accuracy of the model is not predefined [4]. The accuracy of the RSM generated is assumed to be acceptable apriori. An algorithm for developing surrogate models to pre-defined accuracy was developed by Gabbur \& Ramchand is described in [3]. As the accuracy becomes more stringent there would be a reduction of model subspace with concomitant increase in number of iterations. The algorithm creates knowledge database for functions calls and surrogates models. Legacy or historical data if available would also form a part of this knowledge database. This database would reduce the number of times a high fidelity analysis/simulation software tool is run for model generation. The methodology has been been tested on five different optimization test function and the result have been brought out in [3].

\section{Algorithm}

The flow chart for smart RSM is shown in figure 2. The smart RSM comprises of six processes repeated iteratively to generate the validated surrogate models with its design space. The iterative steps are follows

1. Identifying the design space of the model

2. Design of Experiments

3. Analysis of DOE points

4. Generation of Response surface models based on DOE

5. Model Validation

6. Design space reduction

\subsection{Identifying the design space of the model}

The domain of the optimization problem is defined as the initial design space for the surrogate model. Mathematically it can be represented as 
Minimize

$$
\begin{aligned}
& F(\bar{X}) \quad \text { where } \quad(\bar{X}) \in R^{N} \\
& X_{\text {imin }} \leq X_{i} \leq X_{\text {imax }} \quad \text { where } \quad i=1,2,3 \ldots . N
\end{aligned}
$$

Subject to

$$
\begin{aligned}
& \bar{G}(\bar{x}) \geq 0 \text { where } \bar{G} \in R^{m} \\
& \bar{H}(\bar{x})=0 \text { where } \bar{H} \in R^{p}
\end{aligned}
$$

Then the initial design space of the model, for $\mathrm{N}$ dimension, would be defined by equation 4 .

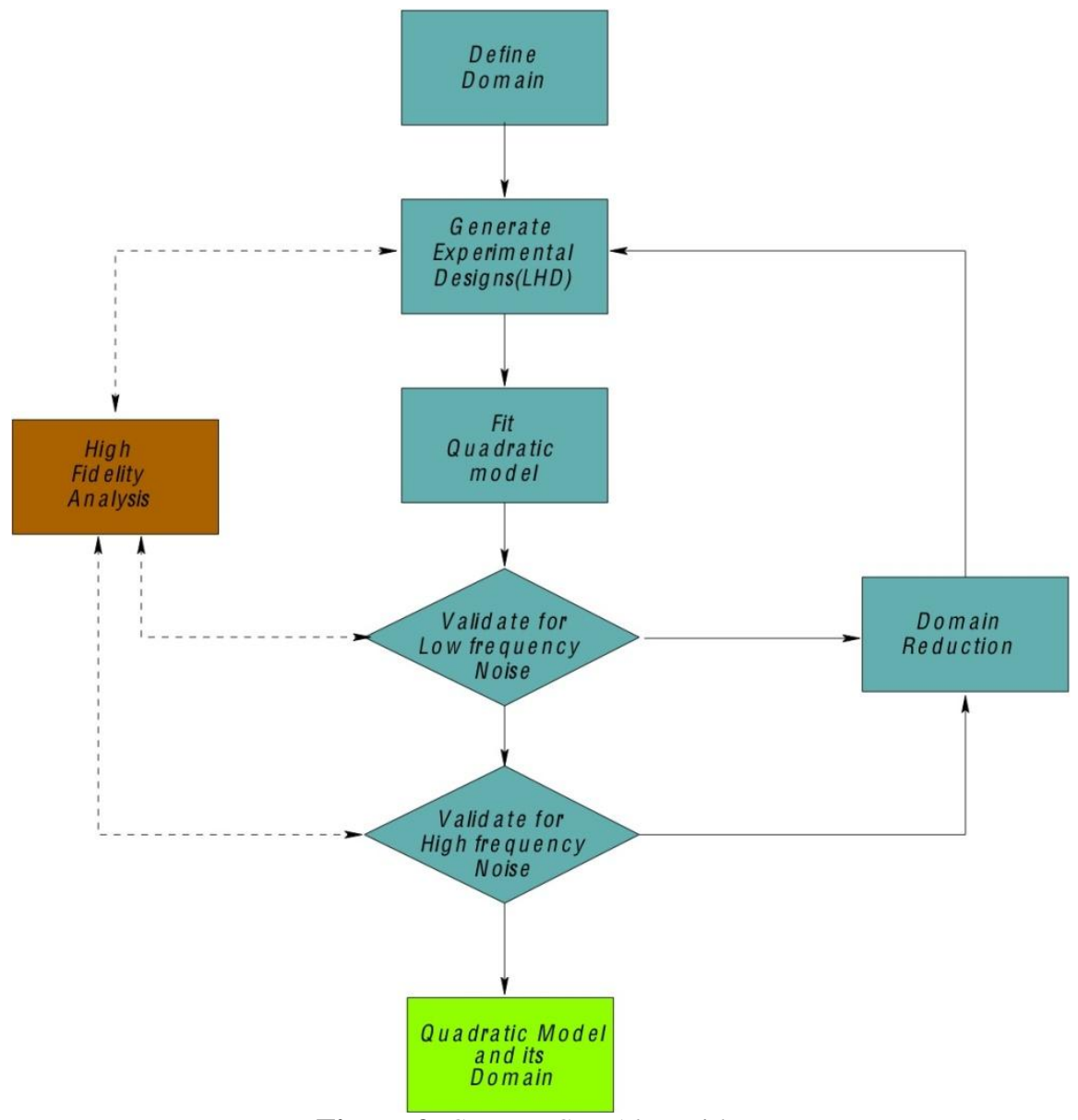

Figure 2: Smart RSM Alogorithm

\subsection{Design of Experiments}

Experimental design techniques, which were initially developed for physical experiments, are _nding considerable use for the design of computer experiments/analyses. In Design of Experiment (DOE) techniques developed for analysis of physical experiments, random variation is accounted for by spreading the sample points out in the design space and by taking multiple data points (replicates). Among various classical experimental designs, Central Composite Design (CCD), alphabetical optimal designs, especially D-optimal designs, are also widely used [8,9]. Sacks, et al. state that the classical techniques of experimental blocking, replication, and randomization are irrelevant when it comes to deterministic computer experiments [10]. Therefore sample points should be chosen to fill the design space for computer experiments. Koch, Mavris and Mistree [11] investigate the use of a modified central composite design (CCD) that combines half-fractions of an inscribed CCD with a face-centered CCD to distribute points more evenly throughout the design space. Koehler and Owen [12] describe several Bayesian space filling.designs, including maximum entropy designs, mean squared-error designs, minimax and maximin designs, Latin Hypercube, randomized orthogonal arrays, and scrambled nets. Widely used space filling sampling methods are Orthogonal Array (OA) and Latin Hyper cube Design (LHD). OA can generate a sample with better space-filling property than LHD. However, the generation of an OA sample is more complicated than LHD [13, 14]. In addition, OA demands strict level 
classification for each variable, which might bring difficulty in real design. In real design, not all combinations of variable level lead to realistic design solutions, and some may cause the crash of the analysis or simulation, which is not uncommon in finite element analysis. In that case, the engineers must manually adjust variables to an appropriate number, deviating from one of the defined levels. Thus the property of OA might be undermined [15]. Therefore for this algorithm LHD is used as DOE method.

\subsection{Analysis of DOE points}

Design analysis is carried out on the points selected by DOE and values of the objective function are evaluated through the computation intensive analysis and simulation processes.

\subsection{Generation of Response surface models based on DOE}

Based on the analysed above design points a quadratic response surface model is fitted to the data using the usual least square method. As an initial test $\mathrm{R}^{2}$ and $\mathrm{R}^{2}$ adjusted are metrics used to estimate and understand the quality of RSM.

\subsection{Model Validation}

The surrogate model is validated for acceptable fit in two stages. The first stage is to check for low frequency errors (gross misfit of the model). This is carried out around a check point in the design space. The check point is generated in such a way that if the model validation fails then the point would be in the new reduced domain. This point is then perturbed for low frequency error. The direction of perturbation is such that the perturbed point also lies in the reduced design space. This is shown in the Figure 3 for a two dimension function.

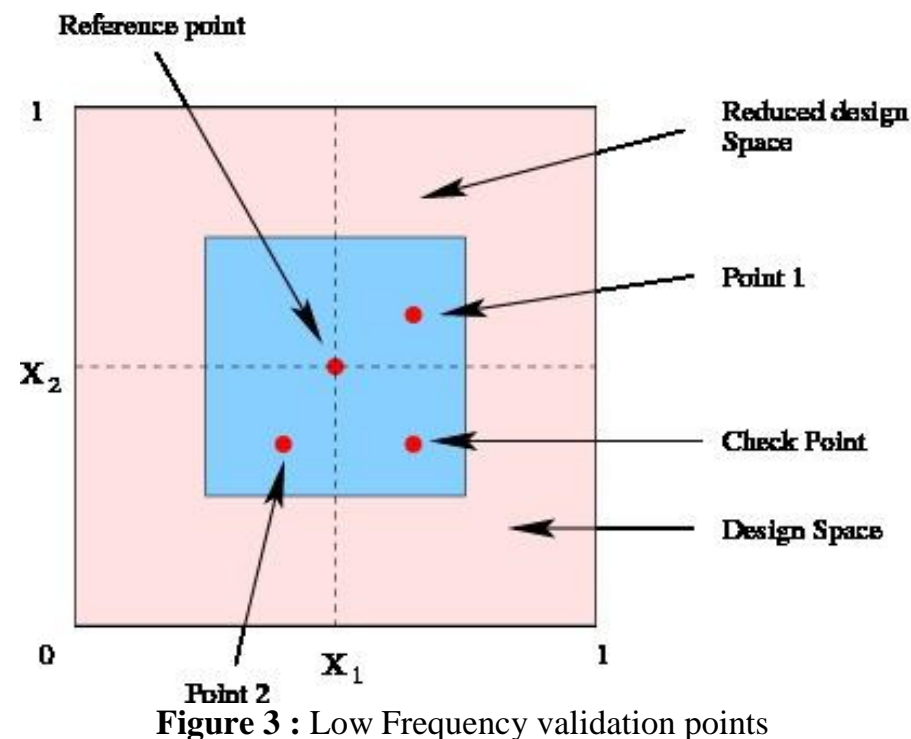

Negative perturbation is given in the $\mathrm{X}_{1}$ direction to get point 1 and positive perturbation is given along $\mathrm{X}_{2}$ to get point 2. The perturbation value $\mathrm{d}$ is $50 \%$ of the reduced domain width for each input. The number of points needed for carrying low frequency error is $(\mathrm{k}+1)$, where $\mathrm{k}$ is the dimension of the input vector. The error (residual) between actual value and the predicted value is calculated at each of these points. This value should be less than predetermined value (typically around 1\%) for the model to be acceptable. Once the model is validated for low frequency then it is checked for high frequency error. For carrying out high frequency validation the above process is repeated with a change in perturbation value. The perturbation value $d$ is changed to $5 \%$ of the reduced design space. The error residuals are calculated as

$$
\begin{aligned}
& \text { Error Residuals }=\frac{Y^{\prime}-Y}{Y} * 100 \\
& \text { where } Y=\text { Actual Value } \\
& Y^{\prime}=\text { Predicted Value }
\end{aligned}
$$




\subsection{Design Space Reduction}

For a complex analysis tool / function a single quadratic model may not satisfactorily represent the analysis tool for the full design space. When surrogate model does not accurately represent the analysis tool then the design space needs to be reduced. A selective reduction of design space is employed. The strategy is to halve the domain for that design variable for which the error residual is more than $1 \%$. Further more design space is reduced (zoomed in) around the reference point. Mathematically $\mathrm{x}_{\mathrm{ri}}$ is the reference point for $\mathrm{i}^{\text {th }}$ input and $\mathrm{x}_{\mathrm{li}}$ and $\mathrm{x}_{\mathrm{ui}}$ are its lower and upper limits respectively then the new design space lower limit $\mathrm{x}_{\mathrm{li}}{ }_{\mathrm{i}}$ and upper limit $\mathrm{x}_{\mathrm{ui}}{ }_{\mathrm{ii}}$ are

$$
\begin{aligned}
& x_{l i}^{\prime}=x_{r i}-\frac{x_{u i}-x_{l i}}{4} \\
& x_{u i}^{\prime}=x_{r i}+\frac{x_{u i}-x_{l i}}{4} \quad \text { for } i=(1,2, \ldots . . k) \\
& \text { if } \quad x_{l i}^{\prime}<x_{l i} \text { then } \\
& x_{l i}^{\prime}=x_{l i} \text { and } x_{u i}^{\prime}=x_{r i}+\frac{x_{u i}-x_{l i}}{2} \\
& \text { if } \quad x_{u i}^{\prime}<x_{u i} \text { then } \\
& x_{u i}^{\prime}=x_{u i} \text { and } x_{l i}^{\prime}=x_{r i}+\frac{x_{u i}-x_{l i}}{2}
\end{aligned}
$$

Figure 4 shows Design space reduction for two dimension design space $(k=2)$. In figure 4(a) and figure 4(b) both the domain are reduced and in figure 4(c) domain for one variable only is reduced. It is proposed to test the algorithm on a higher dimension $\left(10^{\mathrm{d}}\right.$ or higher) realistic design problem. High speed civil transport (HSCT) data is chosen to create a synthetic problem of 25 design variable. The HSCT date used for optimization test problem consists of one objective function and 66 inequality constraints. Noisy functions are created for the objective function and constraints for proving the effectiveness of the smart RSM algorithm in filtering out numerical noise for use in Multidisciplinary Design Optimization.

\section{High Speed Civil Transport (Hsct)}

High speed civil transport (HSCT) is an example of extremely challenging aircraft designs, where the disciplines are highly coupled and results from high fidelity design analysis are critical to establishing the feasibility of the aircraft design. The design concept of HSCT is to fly the aircraft with more than 300 passengers at speeds in excess of 1,500 miles per hour. The aircraft development is by NASA and its industry partners as a next generation supersonic passenger jet of the future [16]. HSCT aircraft configuration is shown in figure 5

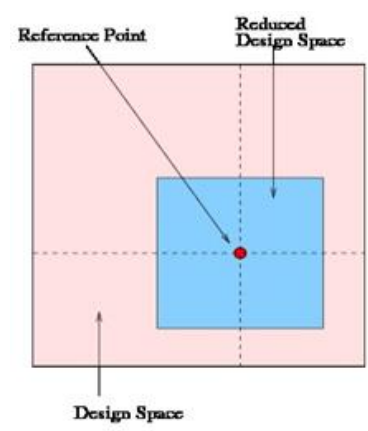

(a)

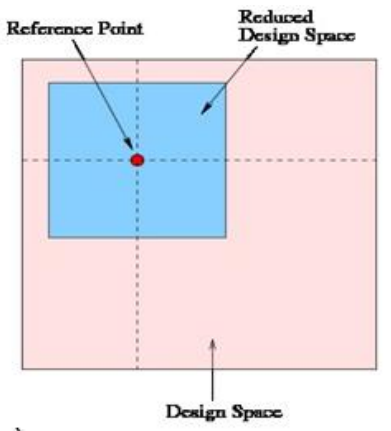

(b)

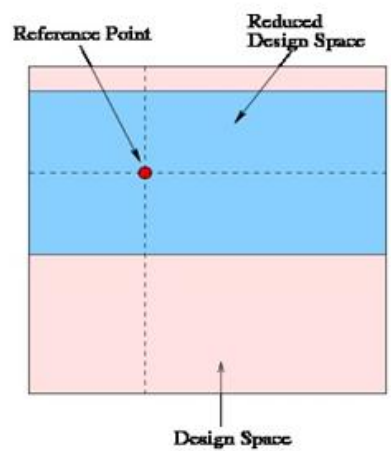

Figure 4: Design Space Reduction 


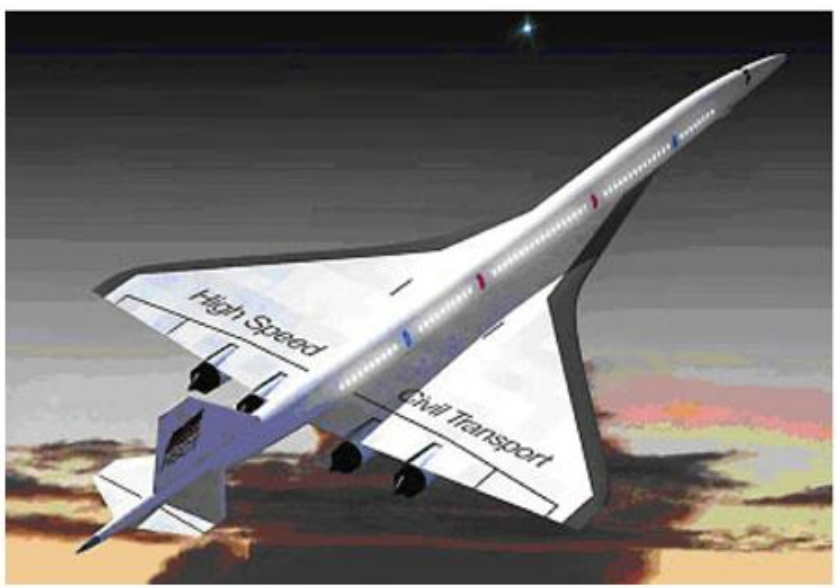

Figure 5 : HSCT Configuration

Multidisciplinary Analysis and Design (MAD) center for advanced vehicles uses HSCT configuration design as a test case for the evaluation of new design optimization methodologies and techniques developed inhouse [17]. The test case is described as minimizing the takeoff gross weight (TOGW) of a High Speed Civil Transport (HSCT) aircraft with range of 5500 nautical mile, designed to cruise at Mach 2.4 and ferry 250 passengers. TOGW was selected as the objective function for optimization problem since it represents a composite measure of merit for the aircraft as a system. TOGW is expressed as a sum of the dry weight (i.e., the weight of the aircraft including payload, but without fuel) and the fuel weight.The dry weight of the aircraft is correlated to the initial acquisition cost of the aircraft and fuel weight represents the yearly recurring costs of aircraft operations [18]. From Multidisciplinary perspective the choice of the gross weight as the objective function incorporates structural and aerodynamic considerations. The structural considerations are directly related to the aircraft empty weight, while the aerodynamic performance dictates the drag and hence the thrust required to overcome the drag which dictates the fuel weight required for the mission. The HSCT design is described by twenty five design variables and sixty eight constraints. Twenty four of these design variables describe the geometry of the aircraft and can be divided into five categories, wing planform, airfoil shape, tail area, nacelle placement and fuselage shape. One variables, mission fuel, defines the cruise mission. Details of the twenty five design variables are given in Table 1.

Table 1 HSCT Design Variables

\begin{tabular}{|l|l|}
\hline Design Variable No & Description \\
\hline 1 & Wing root chord \\
\hline 2 & LE break point, $\mathrm{x}$ \\
\hline 3 & LE break point, $\mathrm{y}$ \\
\hline 4 & TE break point, $\mathrm{x}$ \\
\hline 5 & TE break point, $\mathrm{y}$ \\
\hline 6 & LE wing tip, $\mathrm{x}$ \\
\hline 7 & Wing tip chord \\
\hline 8 & Wing semi-span \\
\hline 9 & Max t/c location \\
\hline 10 & Airfoil t/c at root \\
\hline 11 & Airfoil t/c at LE break \\
\hline 12 & Airfoil t/c at tip \\
\hline 13 & Fuselage restraint $1, \mathrm{x}$ \\
\hline 14 & Fuselage restraint $1, \mathrm{r}$ \\
\hline 15 & Fuselage restraint $2, \mathrm{x}$ \\
\hline 16 & Fuselage restraint 2,r \\
\hline 17 & Fuselage restraint 3,x \\
\hline 18 & Fuselage restraint 3,r \\
\hline 19 & Fuselage restraint 4,x \\
\hline 20 & Fuselage restraint 4,r \\
\hline 21 & Nacelle 1, y \\
\hline 22 & Nacelle 2, y \\
\hline 23 & Mission fuel \\
\hline 24 & Vertical tail area \\
\hline 25 & Horizontal tail area \\
\hline & \\
\hline
\end{tabular}

Sixty eight design constraints define geometry, system performance and aerodynamic performance and are given in Table 2. 
Table 2: HSCT Constraints

\begin{tabular}{|l|l|}
\hline Index & Constraint \\
\hline 1 & Fuel volume $\leq 50$ wing volume \\
\hline 2 & Wing root TE $\leq$ Tail LE \\
\hline $3-20$ & Wing chord $\geq 7.0 \mathrm{ft}$ \\
\hline 21 & LE break within wing semi-span \\
\hline 22 & TE break within wing semi-span \\
\hline 23 & Root chord t/c ratio $\geq 1.5 \%$ \\
\hline 24 & LE break chord t/c ratio $\geq 1.5 \%$ \\
\hline 25 & Tip chord t/c ratio $\geq 1.5 \%$ \\
\hline $26-30$ & Fuselage restraints \\
\hline 31 & Wing spike prevention \\
\hline 32 & Nacelle 1 inboard of nacelle 2 \\
\hline 33 & Nacelle 2 inboard of semi-span \\
\hline 34 & Range $\geq 5500$ nautical miles \\
\hline 35 & CL at landing speed $\leq 1$ \\
\hline $36-53$ & Section CL at landing $\leq 2$ \\
\hline 54 & Landing angle of attack $\leq 12^{\circ}$ \\
\hline $55-58$ & Engine scrape at landing \\
\hline 59 & Wing tip scrape at landing \\
\hline 60 & TE break scrape at landing \\
\hline 61 & Rudder deflection $\leq 22.5^{0}$ \\
\hline 62 & Bank angle at landing $\leq 5^{0}$ \\
\hline 63 & Tail deflection at approach $\leq 22.5^{\circ}$ \\
\hline 64 & Takeoff rotation to occur $\leq$ Vmin \\
\hline 65 & Engine-out limit with vertical tail \\
\hline 66 & Balanced field length $\leq 11000 \mathrm{ft}$ \\
\hline $67-68$ & Mission segments: thrust available $\geq$ thrust required \\
\hline &
\end{tabular}

Multiple configuration of HSCT were analysed over a period of time at NASA Multidisciplinary Analysis and Design (MAD) center for advanced vehicles. The data from this analysis has been collated and is a part of NASA Multidisciplinary Design Optimization Test Suite [17]. It consists of analyses of 2490 HSCT configuration. The data from each analysis is represented in a matrix of 19 rows and 5 columns, and each of the 2,490 matrices is separated by a blank line. The breakup of 95 numbers taken row by row from each $19 \times 5$ matrix is as follows

- Number 1 to Number 25 are the $\mathrm{x}$ vector of 25 design variables which describes each HSCT aircraft configuration. The 25 Design variables are scaled to the order of 1 to 10 .

- $\quad$ Number 26 wing bending material weight

- Number 27 is the takeoff gross weight (TOGW(x)). The objective function, TOGW(x), is not scaled.

- Number 28 to Number 95 represent the sixty eight constraints. The constraints are unscaled and are of order 100-1000 (with negative numbers indicating design infeasibility).

\section{Response Surface Model}

Using the legacy data of HSCT, polynomial based surrogate models (cubic response surface) are created for the objective function and 68 constraints. For generating the response surface models the 68 constraints are scaled. The scaling procedure used for constraints is

Scaled Constraint Value $=($ Maximum value - Actual Value $) /$ Limit

where Limit is defined as difference between maximum value and minimum value of the constraints. The surrogate model is of the form given below

$$
y=\beta_{0}+\sum_{i=1 \text { to } k}^{k} \beta_{i} x_{i}+\sum_{i=1 \text { to } k}^{k} \beta_{i i} x_{i}^{2} \sum_{i=1 \text { to } k}^{k} \beta_{i i i} x_{i}^{3}+\sum_{i=1 \text { to } k}^{k} \sum_{j=i \text { to } k}^{k} \beta_{i j} x_{i} x_{i j}
$$

Latin Hypercube design (LHD) as DOE strategy was used to generate 2000 experimental design points for 25 design variables. The data points nearest to the 2000 experimental design points were selected from the HSCT data and used for generating cubic response surface model. The cubic response surface model is fitted to the data using least square method. For the objective function TOGW, the graph of predicted vs actual value is shown in Figure 6. Residual / error is calculated for the objective function for all 2490 data points. The error is normalised with variance and estimated as follows

$$
\text { Normaised Residuals }=\frac{\text { actual value }- \text { predicted value }}{\text { variance } \sigma}
$$




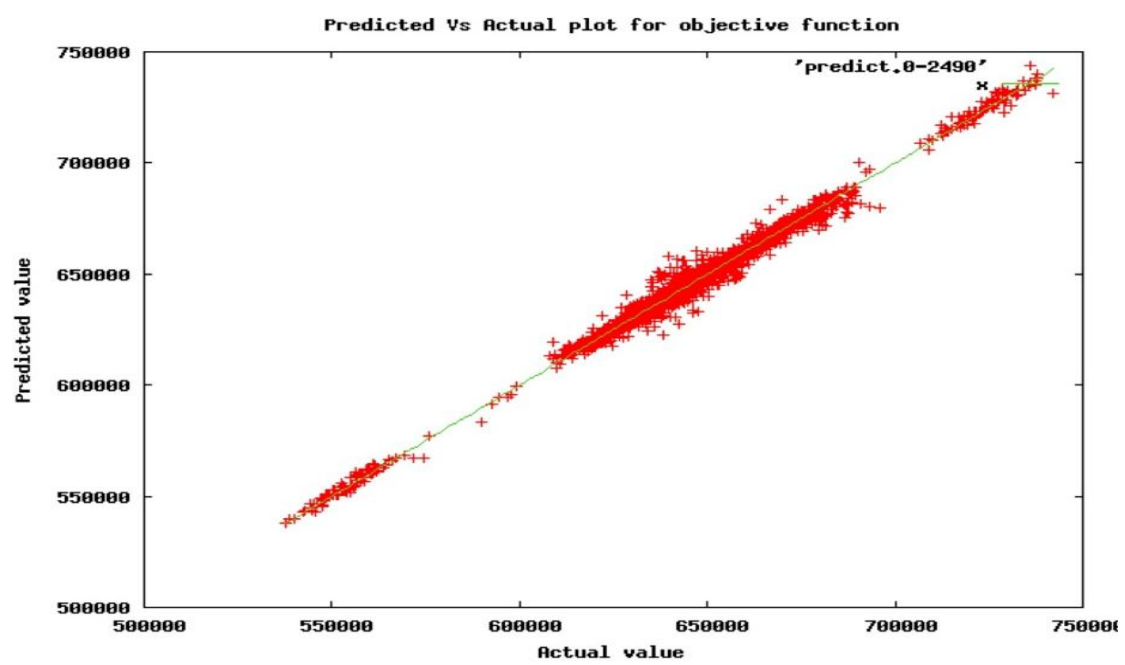

Figure 6 Plot of Objective Function

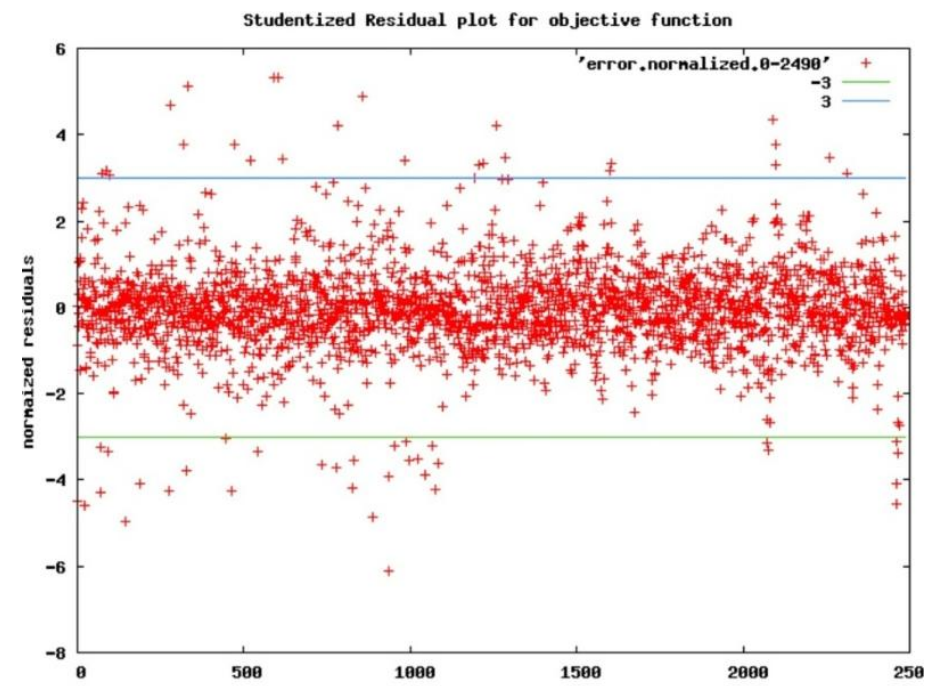

Figure 7 Normalized Residual Error Plot for Objective Functions

The normalised error for the objective function with $+3 \sigma$ and $-3 \sigma \$$ limits is shown in figure 7. Model statistics $\mathrm{R}^{2}$ and $\mathrm{R}_{\text {adjusted }}^{2}$ for objective function are .991093 and 0.982265 respectively. Figure 8 and 9 indicate the spread of $R^{2}$ and $R_{\text {adjusted }}^{2}$ for 66 constraints.

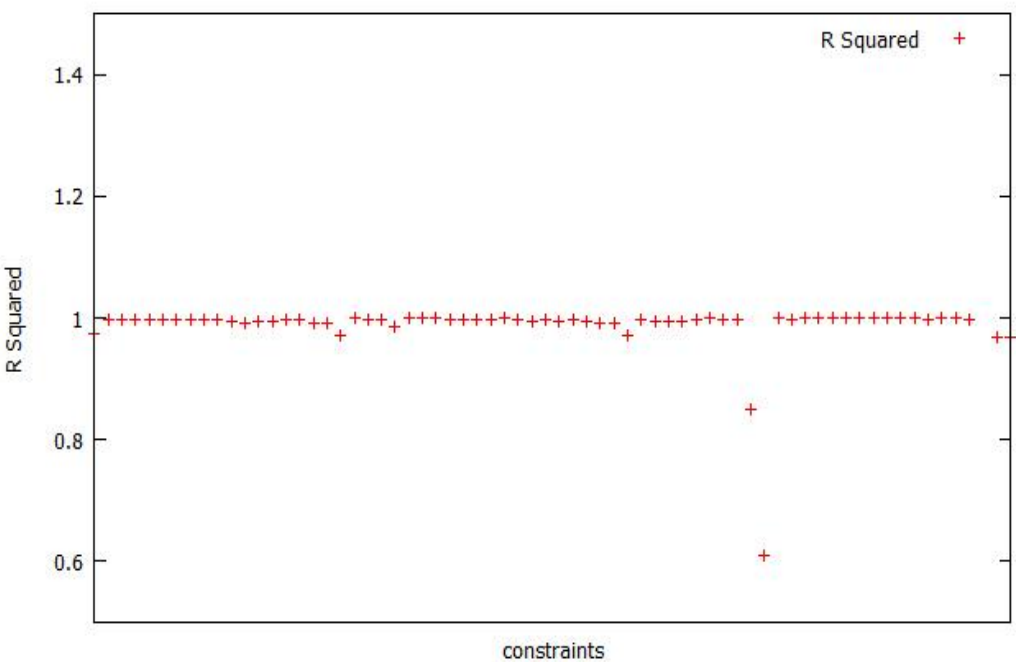

Figure $8: \mathrm{R}^{2}$ for 66 constraints 


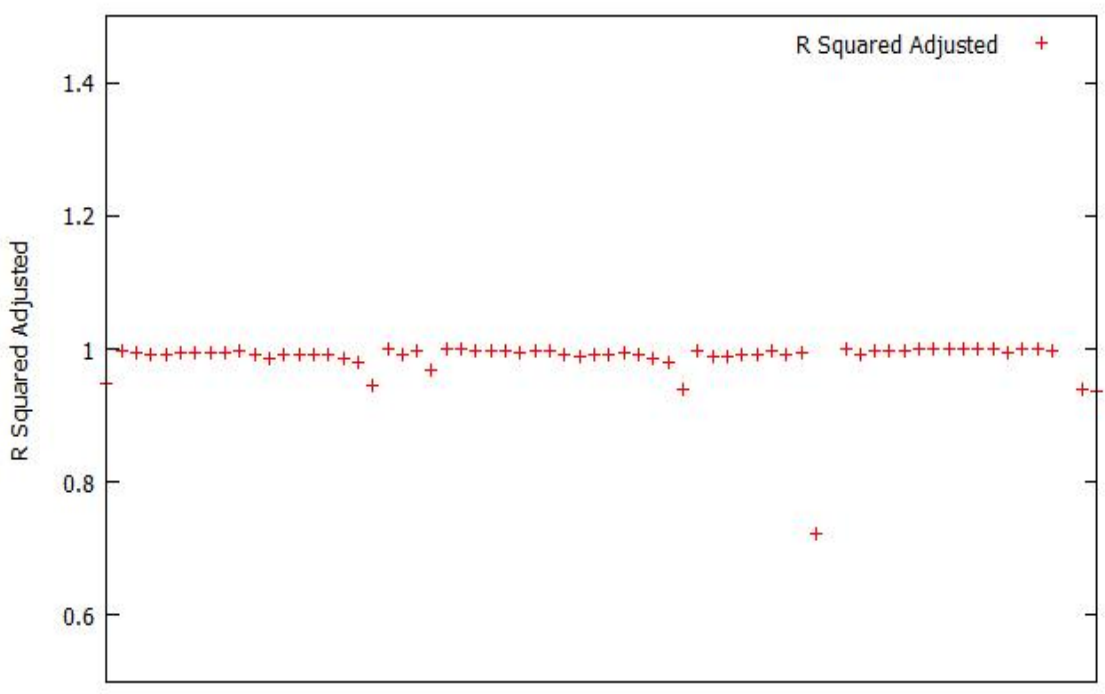

constraints

Figure 9: $\mathrm{R}^{2}$ adjusted for 66 constraints

The error for each model is the characterized using mean and variance $\sigma$. The Error metrics for the objective function and 66 scaled constraints are given in table 3 .

Table 3: Error Metrics for the Surrogate Model

\begin{tabular}{|c|c|c|c|c|c|}
\hline Function & Mean & Sigma & Function & Mean & Sigma \\
\hline Objective & -87.030 & 2956.607 & Constraint 34 & 0.001617 & 0.172839 \\
\hline Constraint 1 & 0.002317 & 0.330295 & Constraint 35 & 0.014505 & 0.221591 \\
\hline Constraint 2 & 0.000351 & 0.009246 & Constraint 36 & 0.010143 & 0.184822 \\
\hline Constraint 3 & 0.001463 & 0.034273 & Constraint 37 & -0.000420 & 0.137855 \\
\hline Constraint 4 & 0.001411 & 0.032661 & Constraint 38 & 0.002242 & 0.140118 \\
\hline Constraint 5 & 0.001360 & 0.032563 & Constraint 39 & 0.004293 & 0.123276 \\
\hline Constraint 6 & 0.001555 & 0.032148 & Constraint 40 & 0.002108 & 0.102640 \\
\hline Constraint 7 & -0.000217 & 0.034550 & Constraint 41 & -0.000411 & 0.013342 \\
\hline Constraint 8 & -0.002202 & 0.039148 & Constraint 42 & -0.000489 & 0.023651 \\
\hline Constraint 9 & -0.002477 & 0.037638 & Constraint 43 & -0.000490 & 0.023634 \\
\hline Constraint 10 & -0.002208 & 0.041348 & Constraint 44 & -0.000430 & 0.025833 \\
\hline Constraint 11 & 0.003470 & 0.042901 & Constraint 45 & -0.000439 & 0.025776 \\
\hline Constraint 12 & 0.004246 & 0.051777 & Constraint 46 & -0.001345 & 0.031570 \\
\hline Constraint 13 & -0.000858 & 0.052123 & Constraint 47 & 0.000000 & 0.000000 \\
\hline Constraint 14 & 0.002668 & 0.057427 & Constraint 48 & -0.011687 & 0.292862 \\
\hline Constraint 15 & 0.001782 & 0.048474 & Constraint 49 & 0.001291 & 0.034009 \\
\hline Constraint 16 & -0.001079 & 0.041063 & Constraint 50 & 0.022865 & 2.302002 \\
\hline Constraint 17 & 0.000149 & 0.047517 & Constraint 51 & -0.000079 & 0.001688 \\
\hline Constraint 18 & 0.001348 & 0.051881 & Constraint 52 & -0.000399 & 0.010767 \\
\hline Constraint 19 & 0.000765 & 0.060724 & Constraint 53 & 0.001689 & 0.025668 \\
\hline Constraint 20 & -0.000000 & 0.000000 & Constraint 54 & -0.000998 & 0.020468 \\
\hline Constraint 21 & -0.000080 & 0.007034 & Constraint 55 & 0.000915 & 0.030134 \\
\hline Constraint 22 & -0.004000 & 0.025713 & Constraint 56 & -0.000000 & 0.000000 \\
\hline Constraint 23 & -0.003393 & 0.069140 & Constraint 57 & 0.000000 & 0.000000 \\
\hline Constraint 24 & -0.000000 & 0.000001 & Constraint 58 & 0.000000 & 0.000000 \\
\hline Constraint 25 & 0.000470 & 0.031493 & Constraint 59 & 0.000000 & 0.000000 \\
\hline Constraint 26 & 0.000926 & 0.063048 & Constraint 60 & -0.000003 & 0.000068 \\
\hline Constraint 27 & 0.002525 & 0.090631 & Constraint 61 & -0.000049 & 0.004281 \\
\hline Constraint 28 & -0.005665 & 0.120384 & Constraint 62 & 0.000024 & 0.045508 \\
\hline Constraint 29 & -0.015115 & 0.154662 & Constraint 63 & -0.000000 & 0.000000 \\
\hline Constraint 30 & -0.017200 & 0.163188 & Constraint 64 & 0.000000 & 0.000000 \\
\hline Constraint 31 & -0.011297 & 0.167016 & Constraint 65 & 0.001646 & 0.050521 \\
\hline Constraint 32 & 0.017417 & 0.168936 & Constraint 66 & -77.501 & 3508.771 \\
\hline Constraint 33 & 0.022139 & 0.193345 & & & \\
\hline
\end{tabular}




\section{Hsct Design Optimization - Test Case}

A synthetic design optimization test problem is created based on available HSCT data. Mathematically optimization test problem of HSCT is stated below

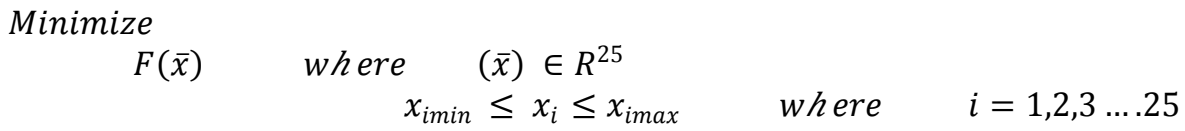

Subject to

$$
G_{j}(\bar{x}) \geq 0 \text { for } j=1,2, \ldots 66
$$

where $F(\bar{x})$ is the take off gross weight, and $\bar{x}$ is the input vector

\section{$G_{j}(\bar{x})$ are the non linearity constraints}

$x_{\min }$ and $x_{\max }$ are the lower and upper bopunds for the design variables

Three optimization problem based on HSCT are defined for validation of Smart Response Surface Models algorithm. The first test case is based on the cubic model (called as smooth function) detailed in section 6. Test case 1 is represented in Figure 10. In the second test case noise is added to the smooth function. The amount of noise added is based on the mean and sigma of the respective model/function. This is represented in Figure 11. In third test case Smart Response Surface Models algorithm is used to generated surrogate models for optimization. The smart RSM interfaces between optimizer and noisy function. The smart RSM generates quadratic models with is move limits and is used by the optimizer. Figure 12 shows interaction between optimizer, smart RSM, noisy function along with RSM and I/O database. It is expected that the Smart RSM would effectively filter numerical noise and optimization would converge with fewer iteration.

The following Nomenclature is used for defining the optimization problem statement

$f_{t}(\bar{x}) \quad$ Cubic fit of weight function to HSCT legacy data set

$\bar{g}_{t}(\bar{x}) \quad$ Cubic $f$ it for constraints to HSCT legacy data set

$f_{n t}(\bar{x}) \quad$ Noisy function of $f_{t}(\bar{x})$

$$
f_{n t}(\bar{x})=f_{t}(\bar{x})+\epsilon \text { where } \epsilon=R N(\mu, \sigma)
$$

$\bar{g}_{n t}(\bar{x}) \quad$ Noisy function of $\left.\bar{g}_{t}(\bar{x})\right)$

$$
\bar{g}_{n t}(\bar{x})=\bar{g}_{t}(\bar{x})+\epsilon \text { where } \epsilon=R N(\mu, \sigma)
$$

$f_{q n}(\bar{x}) \quad$ Quadratic approximation of $f_{n t}(\bar{x})$ with in move limits

$\bar{g}_{q n}(\bar{x}) \quad$ Quadratic approximation of $\bar{g}_{n t}(\bar{x})$ with in move limits

Gradient based optimizer CSFQP is used to solve the three test cases. Gradients are calculated by CSFQP using the built-in finite difference method based function. The stopping criteria for optimizer, $\mathrm{d} 0$ norm, is less than $10^{-}$ ${ }^{6}$. The starting point for optimization, i.e initial design point is a feasible for all the constraints. It is identical for all three test cases.

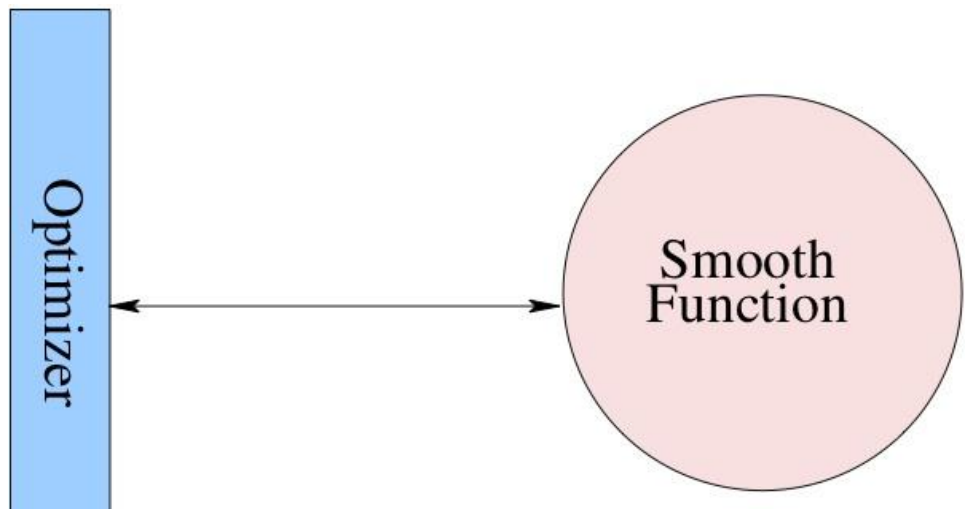

Figure 10: Test Case 1 


\subsection{Test case1}

Problem Statement

\section{Minimize}

$$
\begin{aligned}
& f_{t}(\bar{x}) \quad \text { where }(\bar{x}) \in R^{25} \\
& x_{\text {imin }} \leq x_{i} \leq x_{\text {imax }} \quad \text { where } \quad i=1,2,3 \ldots .25
\end{aligned}
$$

Subject to

$$
\bar{g}_{t}(\bar{x}) \geq 0 \text { where } \quad \bar{g}_{n t} \in R^{66}
$$

CSFQP was run for the above design problem. The starting point for the optimizer was an initial feasible design point. An optimal point was reached after 72 iteration.The d0 norm after 72 iteration was 7.817e -07 . The number of functions calls for by the optimizer for the objective function 1931 . The value of the weight function was 332601.83. This value of the objective function is used as reference value for comparing the other two test cases.

\subsection{Test case 2}

\section{Problem Statement} Minimize

$$
\begin{array}{lcl}
f_{n t}(\bar{x}) & \text { where } & (\bar{x}) \in R^{25} \\
x_{\text {imin }} \leq x_{i} \leq x_{\text {imax }} & \text { where } & i=1,2,3 \ldots .25
\end{array}
$$

Subject to

$$
\bar{g}_{n t}(\bar{x}) \geq 0 \text { where } \bar{g}_{n t} \in R^{66}
$$

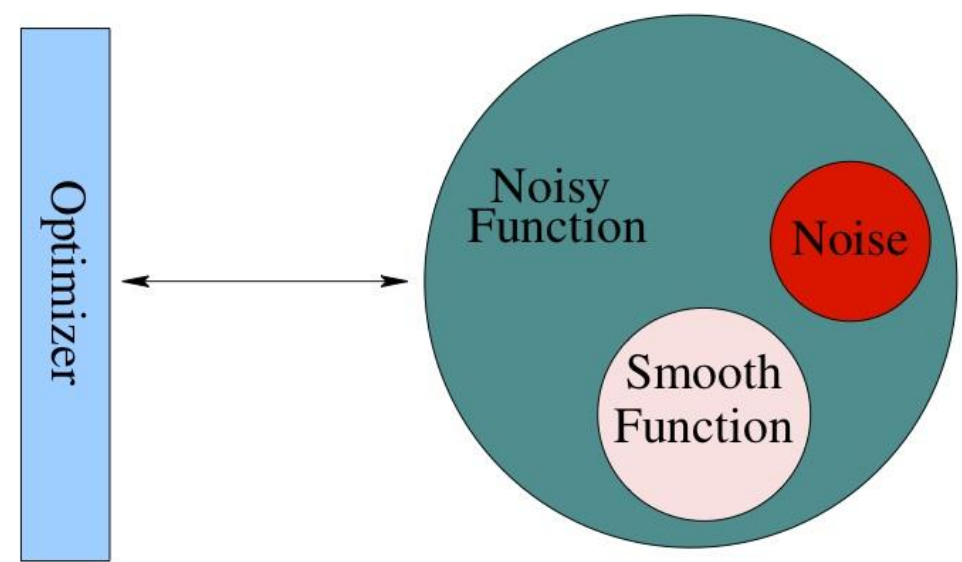

Figure 11: Test Case 2

CSFQP was run for the above design problem with initial design point. The value of objective function at the initial design point is $6.21725 \times 10^{5}$. The optimizer failed to converge after10 iteration. The value of Objective function after10 iteration is 6.21417 times $10^{5}$. The values of the objective function has not reduced much after 10 iteration. The objective function was called 844 times. The function calls to each of 66 constraints was ranging between 840 to 849 . The d0 norm and the step size at the 10 iteration were $3.6058990 \mathrm{e}+00 \mathrm{and}$ $1.4210854 \mathrm{e}-14$ respectively. The optimizer failed as the step size was small. As seen here with a noisy function a gradient based optimizer fails to converge to optima due to numerical noise.

\subsection{Test case 3 - Smart $R S M$}

In test case 3 Smart RSM interfaces between optimizer and the noisy function. It generates validated quadratic RSM (with its subspace) for objective function and 66 constraints to be used by optimizer. The optimization parameters are similar to earlier test cases. The process was repeated twice with two different acceptable modelling error of $10 \backslash \%$ and $5 \backslash \%$. The Smart RSM also interacts with two databases, Input/output and RSM database. At the starting of the optimization process there is no data in both databases during optimization process the databases get populated and values are checked to reuse existing RSM and avoid redundant function calls. 


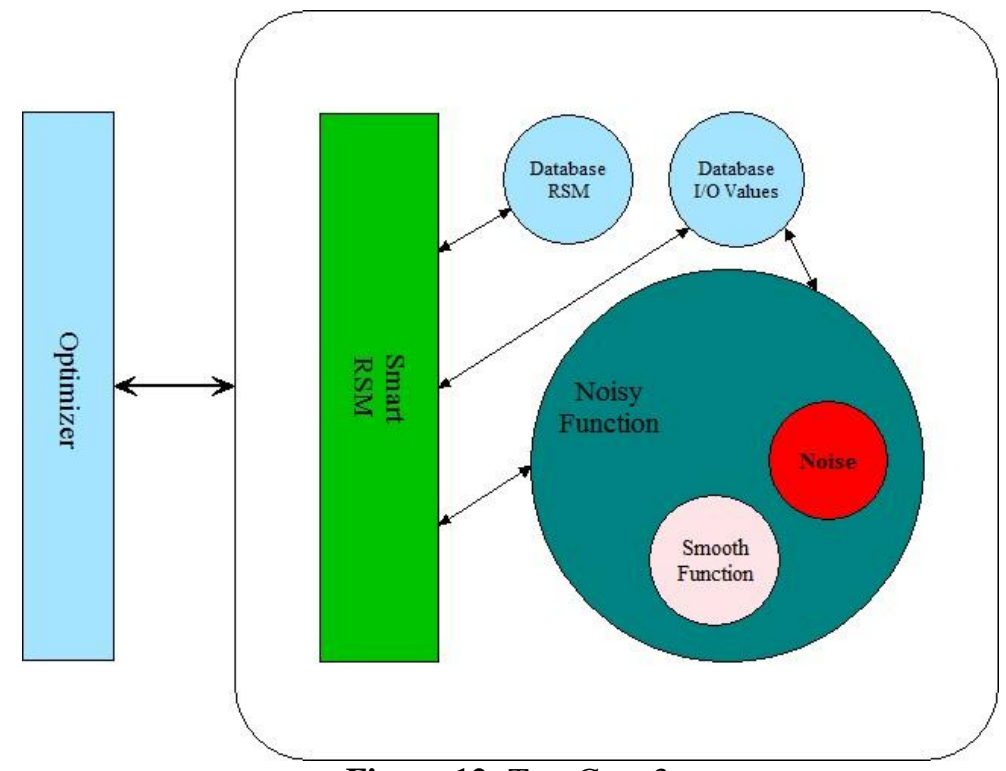

Figure 12: Test Case 3

\begin{tabular}{|c|l|}
\hline Acceptable modelling error & $10 \%$ \\
\hline Number of iterations & 36 \\
\hline d0norm & $2.67651 \times 10^{-7}$ \\
\hline Number of functions calls for objective function & 1636 \\
\hline Objective function $f_{n t}(\bar{x})$ & 374942.05 \\
\hline \multicolumn{1}{|c}{$\%$ error $=\frac{f_{n t}(\bar{x})-f_{t}(\bar{x})}{f_{n t}(\bar{x})} \times 100$} & $12.7 \%$ \\
\hline
\end{tabular}

\begin{tabular}{|l|l|}
\hline Acceptable modelling error & $5 \%$ \\
\hline Number of iterations & 41 \\
\hline d0norm & $9.390395 \times 10^{-7}$ \\
\hline Number of functions calls for objective function & 2426 \\
\hline Objective function $f_{n t}(\bar{x})$ & 366345.560329 \\
\hline \multicolumn{1}{|c}{ error $=\frac{f_{n t}(\bar{x})-f_{t}(\bar{x})}{f_{n t}(\bar{x})} \times 100$} & $10.1 \%$ \\
\hline
\end{tabular}

\section{Result}

Objective function/design analysis tools of the form $f_{t}(\bar{x})$ are smooth and simple functions. Typically these are empirical methods or simple equation used during pre-conceptual design stage. For conceptual/detail design, high fidelity design analysis tools are preferred which are complex and invariably have numerical noise and are similar to $f_{n t}(\bar{x})$. During multidisciplinary optimization process with these design analysis tools, the optimization process either fails due to non- convergence or requires large number of iterations. Smart RSM present a way to overcome these issues.

Table 4 shows the number of iterations, calls to the objective function, and value reached after optimazation for the test cases. Due to the numerical noise introduced in test case 2 the optimization process has failed after 10 iterations without convergence. It is aslo observed that the value of objective function value has not reduced appreciably to $6.21417 \times 10^{5}$ from the initial value of $6.21725 \times 10^{5}$ during optimization process. The number of functions calls by the optimizer for objective function was 844 till non convergence.

Table 4 Test Case Results

\begin{tabular}{|l|l|l|l|l|l|}
\hline & & Iteration & $\begin{array}{l}\text { No Of Objective } \\
\text { Function calls }\end{array}$ & $\begin{array}{l}\text { Objective Function optimal } \\
\text { value }(\mathrm{Kg})\end{array}$ \\
\hline Test Case 1 & & 72 & 1932 & 332061.8 \\
\hline Test Case 2 & & Failed (10) & 844 & 621417.3 \\
\hline Test Case 3 & $10 \%$ & 36 & 1636 & 374942.1 \\
\hline & $5 \%$ & 41 & 2426 & 366345.5 \\
\hline
\end{tabular}

With the implementation of Smart RSM, Test case 3, quadratic approximations with move limits are created and used by optimizer. The number of objective function calls by the smart RSM for an acceptable modelling error of $10 \%$ and $5 \%$ is 1636 and 2426 respectively. For a acceptable modelling error of $10 \%$, Table 
6 gives the details of the function calls for objective functions and 66 constraints. Complex aircraft design has large number of constraints criteria that needs to met. In the case of HSCT there are 66 design constraints. These design constraints are estimated with use of multidisciplinary analysis. This increases the computation efforts and time. With the implementation of Smart RSM on design tools, both for objective function and constraints, there would a saving in computation time and circumventing the problems associated with numerical noise. Further, with appropriate database managements for RSM a design knowledge base could also be developed. Table 5 shows number of models generated for objective function and constraints for $10 \%$ modelling error.

Table 5 : Number of Surrogate models generated

\begin{tabular}{|c|c|c|c|}
\hline Function & RSM created & Function & RSM created \\
\hline Objective & 3 & Constraint 34 & 9 \\
\hline Constraint 1 & 3 & Constraint 35 & 14 \\
\hline Constraint 2 & 2 & Constraint 36 & 11 \\
\hline Constraint 3 & 8 & Constraint 37 & 11 \\
\hline Constraint 4 & 11 & Constraint 38 & 9 \\
\hline Constraint 5 & 12 & Constraint 39 & 11 \\
\hline Constraint 6 & 15 & Constraint 40 & 13 \\
\hline Constraint 7 & 7 & Constraint 41 & 12 \\
\hline Constraint 8 & 14 & Constraint 42 & 7 \\
\hline Constraint 9 & 15 & Constraint 43 & 8 \\
\hline Constraint 10 & 15 & Constraint 44 & 3 \\
\hline Constraint 11 & 14 & Constraint 45 & 3 \\
\hline Constraint 12 & 11 & Constraint 46 & 15 \\
\hline Constraint 13 & 3 & Constraint 47 & 1 \\
\hline Constraint 14 & 16 & Constraint 48 & 3 \\
\hline Constraint 15 & 6 & Constraint 49 & 18 \\
\hline Constraint 16 & 11 & Constraint 50 & 0 \\
\hline Constraint 17 & 3 & Constraint 51 & 3 \\
\hline Constraint 18 & 3 & Constraint 52 & 3 \\
\hline Constraint 19 & 10 & Constraint 53 & 15 \\
\hline Constraint 20 & 1 & Constraint 54 & 20 \\
\hline Constraint 21 & 3 & Constraint 55 & 11 \\
\hline Constraint 22 & 13 & Constraint 56 & 1 \\
\hline Constraint 23 & 11 & Constraint 57 & 1 \\
\hline Constraint 24 & 1 & Constraint 58 & 1 \\
\hline Constraint 25 & 5 & Constraint 59 & 1 \\
\hline Constraint 26 & 12 & Constraint 60 & 1 \\
\hline Constraint 27 & 15 & Constraint 61 & 0 \\
\hline Constraint 28 & 10 & Constraint 62 & 21 \\
\hline Constraint 29 & 9 & Constraint 63 & 1 \\
\hline Constraint 30 & 15 & Constraint 64 & 1 \\
\hline Constraint 31 & 15 & Constraint 65 & 13 \\
\hline Constraint 32 & 14 & Constraint 66 & 0 \\
\hline Constraint 33 & 12 & & \\
\hline
\end{tabular}

Table 6: Function calls details

\begin{tabular}{|l|c|l|l|}
\hline Function & No of calls & Function & No of calls \\
\hline Objective & 1636 & Constraint 34 & 5210 \\
\hline Constraint 1 & 2118 & Constraint 35 & 8067 \\
\hline Constraint 2 & 1117 & Constraint 36 & 6817 \\
\hline Constraint 3 & 5177 & Constraint 37 & 9099 \\
\hline Constraint 4 & 6468 & Constraint 38 & 8972 \\
\hline Constraint 5 & 6549 & Constraint 39 & 9106 \\
\hline Constraint 6 & 9030 & Constraint 40 & 10601 \\
\hline Constraint 7 & 4030 & Constraint 41 & 12949 \\
\hline Constraint 8 & 8795 & Constraint 42 & 6437 \\
\hline Constraint 9 & 9147 & Constraint 43 & 5886 \\
\hline Constraint 10 & 8764 & Constraint 44 & 2227 \\
\hline Constraint 11 & 8672 & Constraint 45 & 2725 \\
\hline Constraint 12 & 6234 & Constraint 46 & 10535 \\
\hline Constraint 13 & 2374 & Constraint 47 & 704 \\
\hline Constraint 14 & 9331 & Constraint 48 & 1643 \\
\hline Constraint 15 & 5832 & Constraint 49 & 13543 \\
\hline Constraint 16 & 8164 & Constraint 50 & 947 \\
\hline Constraint 17 & 2362 & Constraint 51 & 1620 \\
\hline Constraint 18 & 2227 & Constraint 53 & 2227 \\
\hline Constraint 19 & 7206 & Constraint 54 & 8963 \\
\hline Constraint 20 & 704 & & 19637 \\
\hline
\end{tabular}




\begin{tabular}{|l|l|l|l|}
\hline Constraint 21 & 2227 & Constraint 55 & 8428 \\
\hline Constraint 22 & 10189 & Constraint 56 & 704 \\
\hline Constraint 23 & 7195 & Constraint 57 & 704 \\
\hline Constraint 24 & 704 & Constraint 58 & 704 \\
\hline Constraint 25 & 3016 & Constraint 59 & 704 \\
\hline Constraint 26 & 6610 & Constraint 60 & 704 \\
\hline Constraint 27 & 9494 & Constraint 61 & 946 \\
\hline Constraint 28 & 5670 & Constraint 62 & 19843 \\
\hline Constraint 29 & 5105 & Constraint 63 & 704 \\
\hline Constraint 30 & 8986 & Constraint 64 & 704 \\
\hline Constraint 31 & 9786 & Constraint 65 & 8599 \\
\hline Constraint 32 & 7846 & Constraint 66 & 946 \\
\hline Constraint 33 & 7221 & & \\
\hline
\end{tabular}

\section{Acknowledgements}

Authors gratefully acknowledge the support and guidance provided by Dr S. Korthu of Aeronautical Development Agency and Dr K Ramchand of IIAEM, Jain University for this research publication.

\section{References}

[1]. D R Towill.Man-machine interaction in aerospace control systems. The Radio and Electronic Engineer, 50(9):447-458, September 1980.

[2]. M Price, S Raughunathan, and R Curran.An integrated systems engineering approach to aircraft design. Progress In Aerospace Science, 42:331-376, 2006

[3]. Ramesh Gabbur and K Ramchand. Expert systems based response surface models for multidisciplinary design optimization. In Progress in Systems Engineering, volume 1089 of Advances in Intelligent Systems and Computing, pages 527-535. Springer International Publishing, 2015.

[4]. A Giunta A, Dudley J M, Narducci R, Grossman B, Haftka R.T, Mason W H, and Watson L. T. Noisy aerodynamic response and smooth approximations in hsct design. Proceedings of the $5^{\text {th }}$ AIAA/USAF/NASA/ISSMO Symposium on Multidisciplinary Analysis and Optimization, 94(4376):1117-1128, sep 1994.

[5]. Renaud J. E and Gabriele G. A. Improved coordination in non-hierarchical system optimization. AIAA Journal, 31:2367-2373, 1993.

[6]. G. A. Renaud J. Eand Gabriele. Approximation in non-hierarchical system optimization. AIAA Journal, 32:198-205, 1994

[7]. J. C. Korngold and G. A. Gabriele. Multidisciplinary analysis and optimization of discrete problems using response surface methods. Journal of Mechanical Design, 119:427-433, 1997.

[8]. Mitchell T. J. An algorithm for the construction of d-optimal experimental designs. Technometrics, 6-2:203-210, 1997

[9]. R Unal, R. A Lepsch, and M. L McMillin. Response surface model building and multidisciplinary optimization using d-optimal designs. AIAA, 98-4759:405-411, 1998.

[10]. J Sacks, W.J Welch, T.J Mitchel, and H.P Wynn. Design and analysis of computer experimant. Statistical Science, 4:409-435, 1989.

[11]. P. N Koch, D. Mavris, and F Mistree. Multi-level, partitioned response surfaces for modeling complex systems. $7^{\text {th }}$ AIAA/USAF/NASA/ISSMO Symposium on Multidisciplinary Analysis and Optimization AIAA, 4858:1954-1968, 1998.

[12]. J. R. Koehler and A. B Owen. 'Computer Experiments' Handbook of Statistics, volume 261-308. Elsevier Science, New York, 1996.

[13]. G Taguchi, Y Yokoyama, and Wu. Y. Taguchi methods: Design of experiments. American Supplier Institute, Allen Park, Michigan.

[14]. A Owen. Orthogonal arrays for computer experiments, integration, and visualization. StatisticaSinica,, 2:439-452, 1992.

[15]. G. Gary Wang. Adaptive response surface method using inherited latin hypercube design points. ASME, Journal of Mechanical Design, Vol. 125, pp. pp 210-220, June 2003., 125:210-220, June 2003.

[16]. Nasa's high-speed research program.url=http://oea.larc.nasa.gov/PAIS/HSR-Overview2.html.

[17]. Test suite problem $2.1 \mathrm{hsct}$ approximation challenge. utl=http://mdob.larc.nasa.gov/mdo.test/class $2 \mathrm{prob} 1 . h \mathrm{mtml}$.

[18]. Anthony A. Giunta. Aircraft multidisciplinary design Optimization using design of experiments Theory and response surface modeling Methods. PhD thesis, 1997. 